\title{
The soma-germline communication: implications for somatic and reproductive aging
}

\author{
Matthew A. Gaddy ${ }^{1}$ Swana Kuang ${ }^{1}$, Mohammad A. Alfhili ${ }^{2} \&$ Myon Hee Lee ${ }^{1, *}$ \\ ${ }^{1}$ Department of Internal Medicine, Division of Hematology/Oncology, Brody School of Medicine at East Carolina University, Greenville, \\ NC 27834, United States, ${ }^{2}$ Department of Clinical Laboratory Sciences, College of Applied Medical Sciences, King Saud University, \\ Riyadh 11433, Saudi Arabia
}

\begin{abstract}
Aging is characterized by a functional decline in most physiological processes, including alterations in cellular metabolism and defense mechanisms. Increasing evidence suggests that caloric restriction extends longevity and retards age-related diseases at least in part by reducing metabolic rate and oxidative stress in a variety of species, including yeast, worms, flies, and mice. Moreover, recent studies in invertebrates - worms and flies, highlight the intricate interrelation between reproductive longevity and somatic aging (known as disposable soma theory of aging), which appears to be conserved in vertebrates. This review is specifically focused on how the reproductive system modulates somatic aging and vice versa in genetic model systems. Since many signaling pathways governing the aging process are evolutionarily conserved, similar mechanisms may be involved in controlling soma and reproductive aging in vertebrates. [BMB Reports 2021; 54(5): 253-259]
\end{abstract}

\section{INTRODUCTION}

The central role of oxidative damage, telomere attrition, mitochondrial genome damage, and epigenetic alterations in aging is well documented (1). The study of these different causes is best done through the use of model organisms as they are readily amenable to both genetic manipulation and molecular analysis. Two simple model organisms where aging is well studied are yeast (Saccharomyces cerevisiae) and worms (Caenorhabditis elegans) (Fig. 1). The aging of yeast can be monitored in two distinct ways: replicative lifespan and chronological lifespan (2). Replicative lifespan in yeast is determined by the number of divisions that each mother cell can undergo, whereas chronological yeast aging is measured using station-

*Corresponding author. Tel: +1-252-744-3134; Fax: +1-252-744-3418; E-mail: leemy@ecu.edu

https://doi.org/10.5483/BMBRep.2021.54.5.198

Received 14 September 2020, Revised 1 October 2020, Accepted 13 December 2020

Keywords: Aging, Germline, Reproduction, Soma, Soma theory ary phase cultures (2). In C. elegans, lifespan is measured by the amount of average days that the animals live and, as adult worms do not undergo further somatic cell division, aging is post-mitotic. Both of these model organisms have remarkably similar viability curves and mounting evidence indicates common characteristics of aging with mammals (3).

Genetic studies in model organisms have shown that aging is regulated by a specific cluster of genes and have allowed for the analysis of the different pathways involved in physiology, signal transduction, and gene regulation (4). Of note, energy depletion (and calorie restriction) in yeast through glucose deprivation was found to extend the lifespan of mother cells (5). This is due to silencing of DNA via deacetylation of histones by silent information regulator (Sir) proteins (Fig. 1) (4). These proteins are downstream of a signaling pathway involving Sum $1 p$ that detects cellular glucose and responds by repressing key genes that promote aging $(4,6)$. Homologs of the Sir proteins (Sir-2.1) have also been studied in C. elegans where the upregulation of a Sir homolog was able to extend lifespan (7, 8). Like C. elegans, Drosophila melanogaster (D. melanogaster) also exhibits biological properties suitable for aging studies such as high fecundity, short generation time, ease of cultivation, and availability of sophisticated methods for genetic studies (Fig. 1) (9). These attributes also compound with the fact that the role of the insulin/IGF-1 (insulin-like growth factor-1) pathway in aging is evolutionarily conserved (4). Along those lines, findings by Tatar et al. suggest that longevity in $D$. melanogaster is controlled by the ratio of consumed protein relative to carbohydrates (10). This nutrient sensing is thought to occur through insulin-like peptides that regulate longevity. Lifespan extension parallels between C. elegans and D. melanogaster have also been found between $C$. elegans insulin/IGF-1 receptor gene, daf-2 (dauer formation-2), and $D$. melanogaster insulin/IGF-1 receptor, INR (insulin-like receptor) (Fig. 1) (11). In fact, blocking daf-2 expression inhibits PI3K/Akt (Phosphatidylinositol 3-kinase/protein kinase B) pathway, and promotes longevity (12). Very recently, the essential role of INR was highlighted by Ma et al. who showed that probiotics significantly enhance the lifespan of wild-type $D$. melanogaster but does the opposite in InR ${ }^{[\mathrm{E} 19]}$ Drosophila (13).

The mouse model is also an attractive choice for aging studies.

ISSN: 1976-670X (electronic edition)

Copyright (C) 2021 by the The Korean Society for Biochemistry and Molecular Biology

(c) This is an open-access article distributed under the terms of the Creative Commons Attribution Non-Commercial License (http://creativecommons.org/licenses/by-nc/4.0) which permits unrestricted non-commercial use, distribution, and reproduction in any medium, provided the original work is properly cited. 

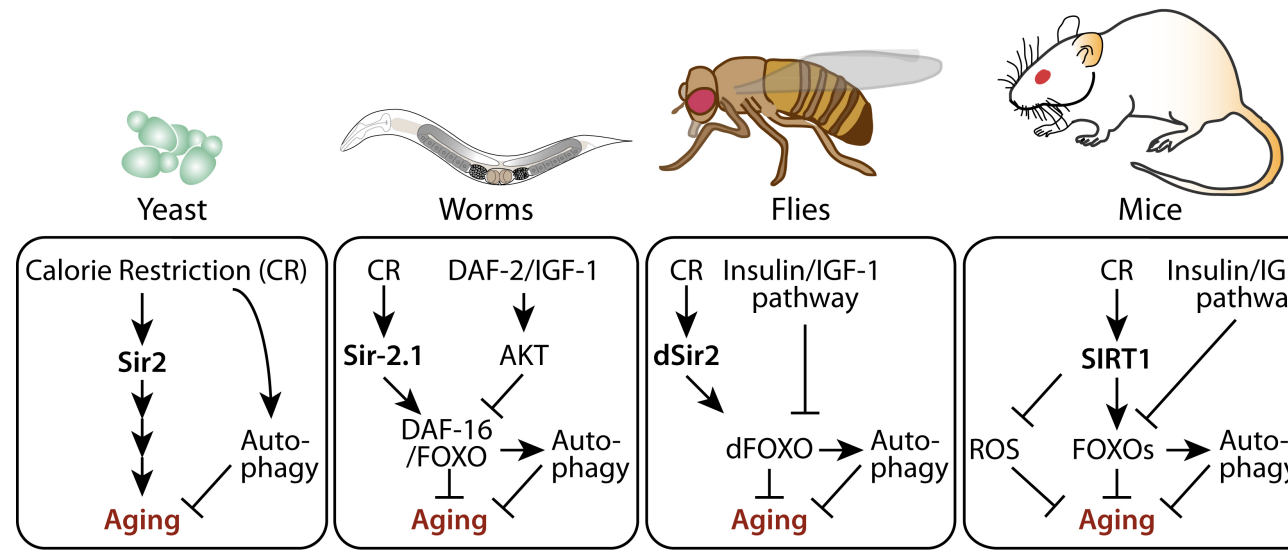

Worms

Flies

Mice
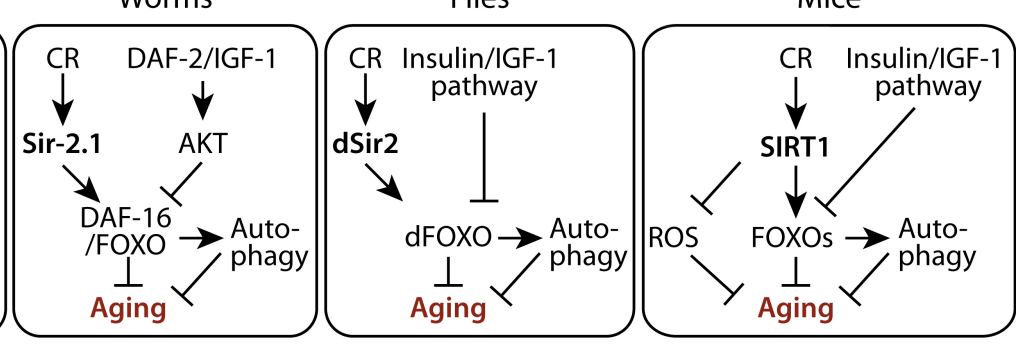

Fig. 1. A conserved pathway for aging in several species. SIR2 homologs (yeast Sir2, worm sir-2.1, fly dSir2, and mouse SIRT1) activated by calorie restriction inhibit aging through FOXO homologs and autophagy. In contrast, Insulin/IGF-1 pathway promotes aging by inhibiting FOXO and autophagy but enhancing oxidative stress.

Mice have a relatively shorter lifespan and a wide array of anatomical and physiological characteristics that are shared with humans (14). In mice, calorie restriction similarly delays aging at least in part through SIRT1-dependent PGC-1 $\alpha$ (peroxisome proliferator-activated receptor gamma coactivator $1 \alpha$ ) activation (15) and SIRT1-mediated antioxidant response in mice (Fig. 1) (16). On the contrary, upregulation of insulin/IGF-1 accelerates aging and predisposes to age-related diseases (Fig. 1) (17). Mice have long telomeres and higher telomerase activity in many organs, which limits the usefulness of comparative studies with humans (14). Using telomerase-deficient mice, it has been demonstrated that telomere damage promotes ageassociated decline in organ function and increased disease risk (18). Moreover, telomere dysfunction accelerates aging in mice and humans, as evidenced by the delay in aging upon experimental stimulation of telomerase in mice (1). However, excessive telomerase activity is a critical step for the development of human cancers. Notably, calorie restriction also attenuates agingassociated shrinkage of telomeres in mouse tissue and reduces the incidence of tumors in mice that overexpress telomerase (19). Therefore, molecular and cellular mechanisms controlling aging and aging-associated pathology are likely conserved across species.

\section{SOMA THEORY OF AGING}

A key principle that describes the trade-off between immortal germline and the disposable soma is Thomas Kirkwood's disposable soma theory of aging. This theory proposes that germline immortality comes at the expense of somatic aging (Fig. 2A and $2 \mathrm{~B})$. Also, the removal of germline delays aging and improves somatic recovery (Fig. 2C) $(20,21)$. Thus, the soma is disposable and the resources are shifted to be used on reproduction, and the germline is labeled as expensive (22). While
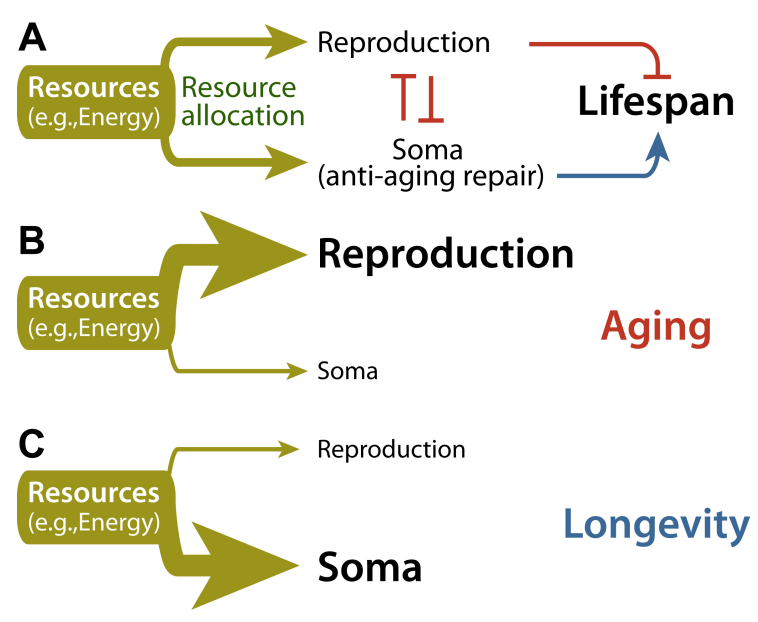

Fig. 2. Communication between reproduction and soma. (A) Disposable soma theory of aging. An organism's lifespan (or aging) may be determined by an evolutionary trade-off between reproduction and tissue maintenance. (B) A greater consumption of a limited amount of resources (e.g., energy) in reproduction may result in reduced investment in tissue maintenance leading somatic aging. (C) Removal of reproductive tissue or reducing its activity delays aging processes in multiple organisms (e.g., Korean eunuchs), including humans.

there has been work supporting this theory, another view challenged the disposable soma theory. Rather than the soma being disregarded so that more resources are focused on germline maintenance, these two components work together through common signaling pathways (22).

The disposable soma theory has been supported through a countless number of experiments using model organisms such as worms, flies, and zebrafish. In regard to C. elegans, studies 
using Catechin and tannic acid demonstrated a decrease in body size in exchange for longevity which argues for the disposable soma theory $(23,24)$. D. melanogaster is another model organism that demonstrates this trade-off, with juvenile hormone $\mathrm{JH})$ as a regulator (25), which will be discussed further in the section on the somatic effect on reproductive aging. In addition, late-reproducing fly strain had a significantly longer lifespan than early-reproducing strain (26). In male zebrafish, the experimental removal of the germline improves somatic recovery in response to stress (Fig. 2C) (21).

As for humans, Min et al. reported the effects of castration and Korean eunuchs on longevity. It was found that the average lifespan of 70 years observed in Korean eunuchs was significantly lowered in non-castrated men who had an average of 50-56 years (27). It was then proposed that having male sex hormones reduced longevity; again supporting the disposable soma theory (27).

\section{REPRODUCTIVE EFFECT ON SOMATIC AGING}

To date, significant progress has been made in understanding genetic and environmental factors of aging. Likewise, the interest in the molecular mechanisms through which the reproductive system modulates somatic aging and vice versa, have recently emerged in multiple model systems. C. elegans have a two-part reproductive system which entails that lifespan is dictated by both germ cells and somatic reproductive tissues (28). C. elegans germ cells are totipotent and constitute the only immortal lineage capable of generating offspring. Somatic cells, however, differentiate into specialized, mortal cell types (29). In order for the germline stem cells (GSC) to achieve immortality, they must exhibit exquisite maintenance and repair machinery (30). In a study by Lee et al., it was found that the germline promotes longevity at $15^{\circ} \mathrm{C}$. Low temperatures are speculated to decrease the damage and deterioration of distinct tissues triggered by cellular metabolism leading to an overall healthier soma (31). Along with this decrease in cellular damage, it is also suggested that the germ cells activate distinct signals to differentially modulate somatic tissues depending on physiological and environmental conditions (18). Uncovering these mechanisms of somatic tissues adopting a more germ cell-like character as well as taking in signals from GSCs may provide a means of dissecting the dichotomy between the instability of somatic cells and the immortality of GSCs (32). Synergistic lifespan extension is observed in a double mutant of DAF-2 (an ortholog of human insulin receptor) and RSKS-1 (an ortholog of human ribosomal protein S6 kinase), which produced nearly a five-fold increase in longevity. In fact, inhibition of RSKS- 1 activates DAF-16 (an ortholog of human FOXO (forkhead box O1)) in the intestine; a master transcriptional regulator involved in stress response against aging. The positive feedback of DAF-16 was mediated through AMP-activated kinase (AMPK) in daf-2 and rsks-1 mutants (33). In a study by Yamawaki et al. (28), the removal of germ cells resulted in nuclear accu- mulation of the transcription factor DAF-16. When germ cells are not removed, DAF-2 has the ability to inhibit DAF-16 by phosphorylation and thus prevent it from nuclear accumulation leading to reduced lifespan. Nuclear accumulation of DAF-16 has also been shown to be stimulated not only by removal of germ cells but also through the use of hydrostatic pressure. Furthermore, Watanabe et al., found cyclic pressure treatment (of $1 \mathrm{MPa}$ once a day for 5 minutes from L1 larvae until death) to significantly increase $C$. elegans lifespan; an event similarly associated with nuclear translocation of DAF-16 (34). Along those lines, it has very recently been demonstrated that DAF-16 does similarly mediate chemically-induced lifespan extension as caused by the terpenoid 3,3-dimethylallyl alcohol (Prenol) (35). This highlights the role of DAF-16 in stress response, adaptive behavior, and regulation of longevity.

Likewise, the effect of germline ablation on longevity was also examined in $D$. melanogaster. The idea of this was based on the study of sterile grandchildless mutants of $D$. subobscura and the finding that such mutants had an extended lifespan (36). GSC- loss is caused by overexpressed $\mathrm{bam}^{+}$(37). The ablation of the germline resulted in an increase in lifespan by $31.3 \%$ to $50 \%$ in females and $21 \%$ to $27.8 \%$ in males (37). Also observed was that germ cell overproliferation shortens the life of the organism (37). Aging delay and increased lifespan was due to the elimination of metabolic demands required to produce gametes, as a result of the removal of the germline (37).

Other studies have focused on intermediate metabolites of the pyrimidine metabolism pathway to infer how it regulates reproductive signals involved in lifespan of $C$. elegans. Extension of lifespan through this pathway, with mediators such as thymine, functions to inhibit reproductive signals and subsequently induce DAF-12 (nuclear receptor of dafachronic acid hormone), NHR (nuclear hormone receptor family), and DAF-16 (38). In another study by Wan et al., it was shown that thymine treatment affected fat metabolism, which may influence aging and longevity. A study by Ratnappan et al. suggests that NHR-49 may facilitate the adaptation to loss of reproductive potential through synchronized enhancement of fatty acid oxidation and desaturation (39). This study also proposes that the conversion of a saturated fatty acid-rich lipid profile to a monounsaturated fatty acid- and polyunsaturated fatty acid-enriched one may lead to enhanced somatic maintenance and lipid signaling, eventually increasing longevity.

Along with lipid metabolism, autophagy is another important lifespan extension mechanism in multiple animals (Fig. 1). Specifically, in C. elegans, germline-loss has also been observed to trigger Target of Rapamycin (TOR) downregulation which stimulates PHA-4 (an ortholog of human Forkhead box A) and autophagy (40). According to a study by Lapierre and Hansen, autophagy was required for the increased lipase activity in animals without germline, which supports the existence of a functional link between autophagy and lipid breakdown (41). As seen with the activation of DAF-16 in the intestine, Lapierre 
and Hansen found that autophagy is predominantly induced in hypodermal and intestinal cells which suggests these tissues are particularly important for longevity. Likewise, the longevitypromoting effects of the flavonoid 5'-Hydroxy-6, 7, 8, 3', 4'pentamethoxyflavone (5-HPF) was through stimulation of autophagy as evident from the increase in transcriptional activity of bec-1 (an ortholog of the human BECN1) and lgg-1 (an ortholog of the human GABA type A receptor associated protein like 1) (42). In congruence with these findings, overexpression of the autophagy receptor SQSTM1 (Sequestosome 1, also called p62) is sufficient to promote longevity in C. elegans and $D$. melanogaster (43). Notably, the conserved function of autophagy in longevity has been proved in multiple animals (Fig. 1).

In a study by Wei and Kenyon, the role of reactive oxygen species (ROS) and hydrogen sulfide in the longevity response to germline loss in $\mathrm{C}$. elegans was examined. With regard to ROS activation, it is thought that germline removal is a signal that germline maturation is incomplete and in response attempts to generate more energy for germline biosynthesis by increasing electron transport rates (44). The hydrogen sulfide pathway was also upregulated by germline-loss and when this pathway was blocked a decrease in longevity in worms without germline was observed (44).

Prostaglandins have also been shown to be involved in longevity and lifespan determination. It was found that a relationship existed between lower, optimal body temperature, germline, and lifespan in C. elegans, as discussed in the study published by Lee et al. (31). The GSCs respond to the decrease in body temperature by releasing prostaglandin E2 (PGE2), which in turn instigates intestinal expression of cbs-1 (cystathionine beta-synthase-1) (31). The intestine then produces more hydrogen sulfide, which may explain the delay in somatic aging in this organism (31). Although colder temperatures are preferred, overexpression of cbs-1 also extends lifespan at warmer temperatures (31).

\section{SOMATIC EFFECT ON REPRODUCTIVE AGING}

In wild-type $C$. elegans, reproduction and aging are inversely related (8). Of note, inhibition of insulin/IGF-1 and TGF- $\beta$ (transforming growth factor- $\beta$ ) Sma/Mab pathways has been shown to slow down reproductive aging. The TGF- $\beta$ Sma/Mab pathway functions in the hypodermis to regulate body size autonomously (45). Intriguingly, Luo et al. have reported that reduction of both pathways attenuates reproductive aging by maintaining oocyte and germline quality non-cell autonomously (46).

DAF-12 is a receptor in C. elegans that impacts lifespan by determining whether induction of reproductive growth, its arrest, or dauer diapause, should occur (47). The arrested state of dauer diapause is chosen in poor growth conditions. 3-kelo bile acid-like steroids, which are comprised of $\Delta 4$-dafachronic acid and $\Delta 7$-dafachronic acids, act on DAF-12 receptor (48). One of influencers for a decision making between dauer diapause and reproductive growth is DAF-9 (an ortholog of human cytochrome P450 family) (48). DAF-9/P450 produces dafachronic acid ligands that activate the DAF-12 nuclear receptor, directing reproductive growth (48-50). Notably, DAF-9 and DAF-12 act through DAF-16 to extend the lifespan of germline-loss worms (48-50).

Studies have shown that there is an inverse relationship between aging and GSC proliferation. As D. melanogaster ages, significant reduction in the rate of GSC proliferation is observed (51). It appears that there are parallel processes that contribute to the maintenance of gametocytes and proliferation of the germline in C. elegans and D. melanogaster. Similar to C. elegans, D. melanogaster also expresses $d p p$ which encodes a protein of the TGF- $\beta$ family that may be involved in reproductive aging if alterations occur such as changes to the GSC niche (52). However, D. melanogaster TGF- $\beta$ is released from the stem cell niche, which is somatic. The expression of this gene in niche cells starting at a young age results in enhanced egg production at later stages of life; it does so by inhibiting differentiation of cystoblasts in germlines (53). Thus, deterioration of reproduction is also influenced by somatic tissues (51). $D$. melanogaster also expresses insulin-like peptide, which is derived from neurosecretory cells located in the brain. This protein normally upregulates GSC proliferation following highprotein intake (54). It has been found that removal of Insulin-like peptides in the brains of young adult female $D$. melanogaster slows down GSC proliferation (55). Additionally, the inhibition of the gonad's insulin receptors delays oogenesis (51). Notably, TGF- $\beta$ signaling has been implicated in multiple aspects of mammalian reproductive aging (56). This suggests that TGF- $\beta$ Sma/Mab and Insulin/IGF-1 are ancestral somatic signaling pathways that govern, at least in part, reproductive aging.

Several studies have been done focusing on $\mathrm{JH}$ and its effects in different invertebrates. $\mathrm{JH}$ is involved in the metamorphosis of this organism by stimulating differentiation by inhibiting the morphogenesis of certain tissues (57). The researchers proposed that $\mathrm{JH}$ was a mediator for reproductive and longevity trade-off (25). There is also an inverse relationship on reproduction, which is caused by $\mathrm{JH}$, and survival (58). This signaling pathway occurs downstream of the Insulin/IGF-1 pathway (25). JH analog $(\mathrm{JHa})$ methoprene was used for selection, to mimic the effects of $\mathrm{JH}$-deficiency in flies. As expected, $\mathrm{JHa}$ treatment increased mortality in the unselected, susceptible controls in exchange for early fertility (25). Upon successful selection, flies gained resistance to $\mathrm{JHa}$, extending their lifespan by an average of 3.8 days (25). This finding was in agreement with the longer lifespan in flies without JH (25).

It is established that hormones are essential in regulating body processes, and germline aging is no exception. The anterior pituitary gland is a major organ of the endocrine system that secretes gonadotropins, such as luteinizing hormone $(\mathrm{LH})$. LH plays an important role in reproductive aging in mammals (59). A preovulatory LH surge causes oocytes to be arrested during prophase I of meiosis in females. Likewise, $\mathrm{LH}$ also 
increases the survival rate of male germ cells (60). In a study by Kawamura et al., a regulatory mechanism controlled by the anterior pituitary gland of Rattus norvegicus was demonstrated. In particular, activation of the Gi pathway caused significant reduction in cAMP (cyclic adenosine monophosphate) levels (60). Additionally, administration of Leydig insulin-like 3 (INSL3) suppressed programmed cell death in male germ cells and prevented the arrest of oocytes at prophase I (60). Furthermore, glial cell line-derived neurotrophic factor (GDNF) is released from somatic Sertoli cells of Mus musculus to influence selfrenewal, differentiation, and maintenance of GSCs, as demonstrated by premature depletion of GSCs in gdnf ${ }^{+/-}$mice (61). Other signals originating from the Sertoli cells are bone morphogenetic protein 4 (BMP4) and stem cell factor (SCF) which contribute to germ cell maintenance and differentiation, respectively (62).

\section{CONCLUSION}

Mounting evidence suggests that somatic tissues play a critical role in reproductive aging and vice versa. Using animal models such as C. elegans, D. melanogaster, $R$. norvegicus, and M. musculus, the interaction between somatic tissue and germline can be extrapolated to other organisms, including humans. It was also widely accepted that organisms allocate limited resources (e.g., energy) to produce either more offspring (reproduction) or to live longer (soma tissue maintenance). The disposable soma theory of aging proposed by Kirkwood states that it is evolutionarily beneficial to sacrifice the soma tissue maintenance (longevity) for the sake of the reproduction. Although many animal models including humans appear to support this theory, it is still controversial (63) - Ermolaeva et al. found that a systemic response to DNA damage in germ cells protects somatic tissues against multiple stresses (64). Therefore, we suggest that the soma-germline communication on aging may be a unidirectional and a bidirectional, depending on external and intrinsic conditions.

\section{ACKNOWLEDGEMENTS}

We thank members of the Lee laboratory and ECU Reproductive Biology Interest Group (RBIG) for insightful discussions. We are also grateful to Jiwoo Lee (Hope Middle School, NC, USA) and Jiah Lee (Hope Middle School, NC, USA) for assistance with figures. This work was supported by NIA (AG06037301) to MHL.

\section{CONFLICTS OF INTEREST}

The authors have no conflicting interests.

\section{REFERENCES}

1. Lopez-Otin C, Blasco MA, Partridge L, Serrano M and
Kroemer G (2013) The hallmarks of aging. Cell 153, 11941217

2. Tissenbaum HA and Guarente $L$ (2002) Model organisms as a guide to mammalian aging. Dev Cell 2, 9-19

3. Troen BR (2003) The biology of aging. Mt Sinai J Med 70, 3-22

4. Guarente L and Kenyon C (2000) Genetic pathways that regulate ageing in model organisms. Nature 408, 255-262

5. Lin SJ, Defossez PA and Guarente L (2000) Requirement of NAD and SIR2 for life-span extension by calorie restriction in Saccharomyces cerevisiae. Science 289, 21262128

6. Higuchi-Sanabria R, Vevea JD, Charalel JK, Sapar ML and Pon LA (2016) The transcriptional repressor Sum1p counteracts Sir2p in regulation of the actin cytoskeleton, mitochondrial quality control and replicative lifespan in Saccharomyces cerevisiae. Microb Cell 3, 79-88

7. Tissenbaum HA and Guarente L (2001) Increased dosage of a sir-2 gene extends lifespan in Caenorhabditis elegans. Nature 410, 227-230

8. Yoon DS, Cha DS, Choi Y, Lee JW and Lee MH (2019) MPK-1/ERK is required for the full activity of resveratrol in extended lifespan and reproduction. Aging Cell 18, e12867

9. Aigaki T, Seong KH and Matsuo T (2002) Longevity determination genes in Drosophila melanogaster. Mech Ageing Dev 123, 1531-1541

10. Tatar M, Post S and Yu K (2014) Nutrient control of Drosophila longevity. Trends Endocrinol Metab 25, 509-517

11. Clancy DJ, Gems D, Harshman LG et al (2001) Extension of life-span by loss of CHICO, a Drosophila insulin receptor substrate protein. Science 292, 104-106

12. Bao J, Liu B and Wu C (2020) Progress of anti-aging drugs targeting autophagy. Adv Exp Med Biol 1207, 681-688

13. Ma S, Sun H, Yang W, Gao M and Xu H (2020) Impact of probiotic combination in InR([E19])/TM2 Drosophila melanogaster on longevity, related gene expression, and intestinal microbiota: a preliminary study. Microorganisms 8 , 1027, 1-14

14. Vanhooren $V$ and Libert $C$ (2013) The mouse as a model organism in aging research: usefulness, pitfalls and possibilities. Ageing Res Rev 12, 8-21

15. Higashida K, Kim SH, Jung SR, Asaka M, Holloszy JO and Han DH (2013) Effects of resveratrol and SIRT1 on PGC1alpha activity and mitochondrial biogenesis: a reevaluation. PLoS Biol 11, e1001603

16. Guarente $L$ (2013) Calorie restriction and sirtuins revisited. Genes Dev 27, 2072-2085

17. Junnila RK, List EO, Berryman DE, Murrey JW and Kopchick JJ (2013) The GH/IGF-1 axis in ageing and longevity. Nat Rev Endocrinol 9, 366-376

18. Jaskelioff M, Muller FL, Paik JH et al (2011) Telomerase reactivation reverses tissue degeneration in aged telomerasedeficient mice. Nature 469, 102-106

19. Vera $E$, Bernardes de Jesus $B$, Foronda $M$, Flores JM and Blasco MA (2013) Telomerase reverse transcriptase synergizes with calorie restriction to increase health span and extend mouse longevity. PLoS One 8, e53760

20. Kirkwood TB (1977) Evolution of ageing. Nature 270, 301-304

21. Chen HY, Jolly C, Bublys K, Marcu D and Immler S 
(2020) Trade-off between somatic and germline repair in a vertebrate supports the expensive germ line hypothesis. Proc Natl Acad Sci U S A 117, 8973-8979

22. Maklakov AA and Immler S (2016) The expensive germline and the evolution of ageing. Curr Biol 26, R577-R586

23. Saul N, Pietsch K, Menzel R, Sturzenbaum SR and Steinberg CE (2010) The longevity effect of tannic acid in Caenorhabditis elegans: Disposable Soma meets hormesis. J Gerontol A Biol Sci Med Sci 65, 626-635

24. Saul N, Pietsch K, Menzel R, Sturzenbaum SR and Steinberg CE (2009) Catechin induced longevity in C. elegans: from key regulator genes to disposable soma. Mech Ageing Dev 130, 477-486

25. Flatt T and Kawecki TJ (2007) Juvenile hormone as a regulator of the trade-off between reproduction and life span in Drosophila melanogaster. Evolution 61, 1980-1991

26. Sgro CM and Partridge L (1999) A delayed wave of death from reproduction in Drosophila. Science 286, 2521-2524

27. Min KJ, Lee CK and Park HN (2012) The lifespan of Korean eunuchs. Curr Biol 22, R792-793

28. Yamawaki TM, Arantes-Oliveira N, Berman JR, Zhang P and Kenyon C (2008) Distinct activities of the germline and somatic reproductive tissues in the regulation of Caenorhabditis elegans' longevity. Genetics 178, 513-526

29. Knutson AK, Rechtsteiner A and Strome $S$ (2016) Reevaluation of whether a soma-to-germ-line transformation extends lifespan in Caenorhabditis elegans. Proc Natl Acad Sci U S A 113, 3591-3596

30. Wang D, Hou L, Nakamura S et al (2017) LIN-28 balances longevity and germline stem cell number in Caenorhabditis elegans through let-7/AKT/DAF-16 axis. Aging Cell $16,113-124$

31. Lee HJ, Noormohammadi A, Koyuncu S et al (2019) Prostaglandin signals from adult germ stem cells delay somatic aging of Caenorhabditis elegans. Nat Metab 1, 790-810

32. Curran SP, Wu X, Riedel CG and Ruvkun G (2009) A soma-to-germline transformation in long-lived Caenorhabditis elegans mutants. Nature 459, 1079-1084

33. Chen D, Li PW, Goldstein BA et al (2013) Germline signaling mediates the synergistically prolonged longevity produced by double mutations in daf- 2 and rsks- 1 in C. elegans. Cell Rep 5, 1600-1610

34. Watanabe N, Morimatsu M, Fujita A et al (2020) Increased hydrostatic pressure induces nuclear translocation of DAF16/FOXO in C. elegans. Biochem Biophys Res Commun 523, 853-858

35. Phulara SC, Pandey S, Jha A, Chauhan PS, Gupta P and Shukla V (2020) Hemiterpene compound, 3,3-dimethylallyl alcohol promotes longevity and neuroprotection in Caenorhabditis elegans. Geroscience doi: 10.1007/s11357-02000241-w [Online ahead of print]

36. Flatt T (2011) Survival costs of reproduction in Drosophila. Exp Gerontol 46, 369-375

37. Flatt T, Min KJ, D'Alterio C et al (2008) Drosophila germ-line modulation of insulin signaling and lifespan. Proc Natl Acad Sci U S A 105, 6368-6373

38. Wan QL, Meng X, Fu X et al (2019) Intermediate metabolites of the pyrimidine metabolism pathway extend the lifespan of $C$. elegans through regulating reproductive sig- nals. Aging (Albany NY) 11, 3993-4010

39. Ratnappan R, Amrit FR, Chen SW et al (2014) Germline signals deploy NHR-49 to modulate fatty-acid beta-oxidation and desaturation in somatic tissues of $\mathrm{C}$. elegans. PLoS Genet 10, e1004829

40. Antebi A (2013) Regulation of longevity by the reproductive system. Exp Gerontol 48, 596-602

41. Lapierre LR and Hansen M (2012) Lessons from C. elegans: signaling pathways for longevity. Trends Endocrinol Metab 23, 637-644

42. Trivedi S and Pandey R (2020) 5'-Hydroxy-6, 7, 8, 3', 4'-pentamethoxyflavone extends longevity mediated by DR-induced autophagy and oxidative stress resistance in C. elegans. Geroscience doi: 10.1007/s11357-020-002296 [Online ahead of print]

43. Aparicio R, Hansen M, Walker DW and Kumsta C (2020) The selective autophagy receptor SQSTM1/p62 improves lifespan and proteostasis in an evolutionarily conserved manner. Autophagy 16, 772-774

44. Wei Y and Kenyon C (2016) Roles for ROS and hydrogen sulfide in the longevity response to germline loss in Caenorhabditis elegans. Proc Natl Acad Sci U S A 113, E28322841

45. Wang J, Tokarz R and Savage-Dunn C (2002) The expression of TGFbeta signal transducers in the hypodermis regulates body size in C. elegans. Development 129, 49894998

46. Luo S, Kleemann GA, Ashraf JM, Shaw WM and Murphy CT (2010) TGF-beta and insulin signaling regulate reproductive aging via oocyte and germline quality maintenance. Cell 143, 299-312

47. Antebi A, Culotti JG and Hedgecock EM (1998) daf-12 regulates developmental age and the dauer alternative in Caenorhabditis elegans. Development 125, 1191-1205

48. Motola DL, Cummins CL, Rottiers V et al (2006) Identification of ligands for DAF-12 that govern dauer formation and reproduction in C. elegans. Cell 124, 1209-1223

49. Berman JR and Kenyon C (2006) Germ-cell loss extends C. elegans life span through regulation of DAF-16 by kri-1 and lipophilic-hormone signaling. Cell 124, 1055-1068

50. Beckstead RB and Thummel CS (2006) Indicted: worms caught using steroids. Cell 124, 1137-1140

51. Tatar M (2010) Reproductive aging in invertebrate genetic models. Ann N Y Acad Sci 1204, 149-155

52. Pan L, Chen S, Weng C et al (2007) Stem cell aging is controlled both intrinsically and extrinsically in the Drosophila ovary. Cell Stem Cell 1, 458-469

53. Waskar M, Li Y and Tower J (2005) Stem cell aging in the Drosophila ovary. Age (Dordr) 27, 201-212

54. Hsu HJ, LaFever L and Drummond-Barbosa D (2008) Diet controls normal and tumorous germline stem cells via insulin-dependent and -independent mechanisms in Drosophila. Dev Biol 313, 700-712

55. LaFever L and Drummond-Barbosa D (2005) Direct control of germline stem cell division and cyst growth by neural insulin in Drosophila. Science 309, 1071-1073

56. Hamatani T, Falco G, Carter MG et al (2004) Age-associated alteration of gene expression patterns in mouse oocytes. Hum Mol Genet 13, 2263-2278

57. Truman JW and Riddiford LM (2002) Endocrine insights 
into the evolution of metamorphosis in insects. Annu Rev Entomol 47, 467-500

58. Flatt T, Tu MP and Tatar M (2005) Hormonal pleiotropy and the juvenile hormone regulation of Drosophila development and life history. Bioessays 27, 999-1010

59. Rubin BS (2000) Hypothalamic alterations and reproductive aging in female rats: evidence of altered luteinizing hormone-releasing hormone neuronal function. Biol Reprod 63, 968-976

60. Kawamura K, Kumagai J, Sudo S et al (2004) Paracrine regulation of mammalian oocyte maturation and male germ cell survival. Proc Natl Acad Sci U S A 101, 7323-
7328

61. Meng X, Lindahl M, Hyvonen ME et al (2000) Regulation of cell fate decision of undifferentiated spermatogonia by GDNF. Science 287, 1489-1493

62. Wong MD, Jin Z and Xie T (2005) Molecular mechanisms of germline stem cell regulation. Annu Rev Genet 39, 173-195

63. Douglas PM and Dillin A (2014) The disposable soma theory of aging in reverse. Cell Res 24, 7-8

64. Ermolaeva MA, Segref A, Dakhovnik A et al (2013) DNA damage in germ cells induces an innate immune response that triggers systemic stress resistance. Nature 501, 416-420 